\title{
SOME CONSIDERATIONS ABOUT FORCES AND DEFORMATIONS IN TIRES
}

\author{
Ronaldo de Breyne Salvagni \\ University of Sao Paulo, Escola Politecnica \\ E-mail: salvagni@usp.br
}

\begin{abstract}
The existing tire models are basically of three kinds: essentially empirical ("magic formulas"), or mixed empirical/analytical, or extremely complex theoretical models almost useless in practical situations. The model here proposed does not require any empirical data, and presents a simple theoretical approach very suitable to use in project and analysis of real suspension systems. This paper suggests a physical and mathematical model for the mechanical behavior of pneumatic car tires, based on the relationship between the relative displacement of the wheel to the tire-ground contact region.

This formulation, assuming small displacements, is the first step to a more comprehensive model of the tire dynamic behavior, which will be published later.
\end{abstract}

\section{INTRODUCTION}

The tire is the structural vehicle component that exchange forces and moments with road surface to support and control vehicle attitude. Ways to deal with the rolling contact phenomena are relevant tools for vehicle dynamics engineer and the tire designer. It is usual to formulate empirical expression based on experimental data to describe tire behavior. This approach does not have any physical or mechanical fundamental relationship with the tire structure itself. Several models with different approach, purpose, level of complexity and accuracy have been proposed by various authors during last half century, accessing only partial aspects of this complex system. Finite element method (NAKASHIMA, et al., 1993), multi radial spoke model or brush model (SHARP, et al., 1986), (Duggof, Fancher, Segel, 1970, Pacejka, 1972), flexible ring model (Miège, 2004), membrane model (ZACHOW, 1997), shell model (LECOMTE, et al., 2010), modal synthesis method (Guan, 1999, Shang 2002), experimental data multiparametric polynomial interpolation (Bakker,(Delft) 1987, Segel et el (UMTRI Michigan) 1977, Pacejka (Delft), 1993) are some examples.

In this text, the mechanical behavior of an inflated tire will be represented by a structural model called "membrane", or "thin walled involucres" (TIMOSHENKO, et al., 1970), (PISSARENKO, et al., 1985), which physical and mathematical models will be presented in the next item. The interface region between the tire and the road will be focused, with the goal of obtaining a detailed distribution of the forces systems acting in this region. The appropriate integration of these forces systems will give the values of the forces and moments acting at the vehicle suspension system. 
This model is essentially different from those presented by several authors (for instance, (BÖHM, 1966), (VIL'KE, et al., 1998), (VIL'KE, et al., 2001), (VIL'KE, et al., 2004), (PACEJKA, 2006)).

This paper presents the general linearized relationship between displacements and forces on tires. The formulation of the referred coefficients will be published soon.

\section{PHYSICAL MODEL}

\subsection{Some facts about tires}

An actual wheel for passenger cars is shown in Figure 1-1, including a cross section with its elements nomenclature.
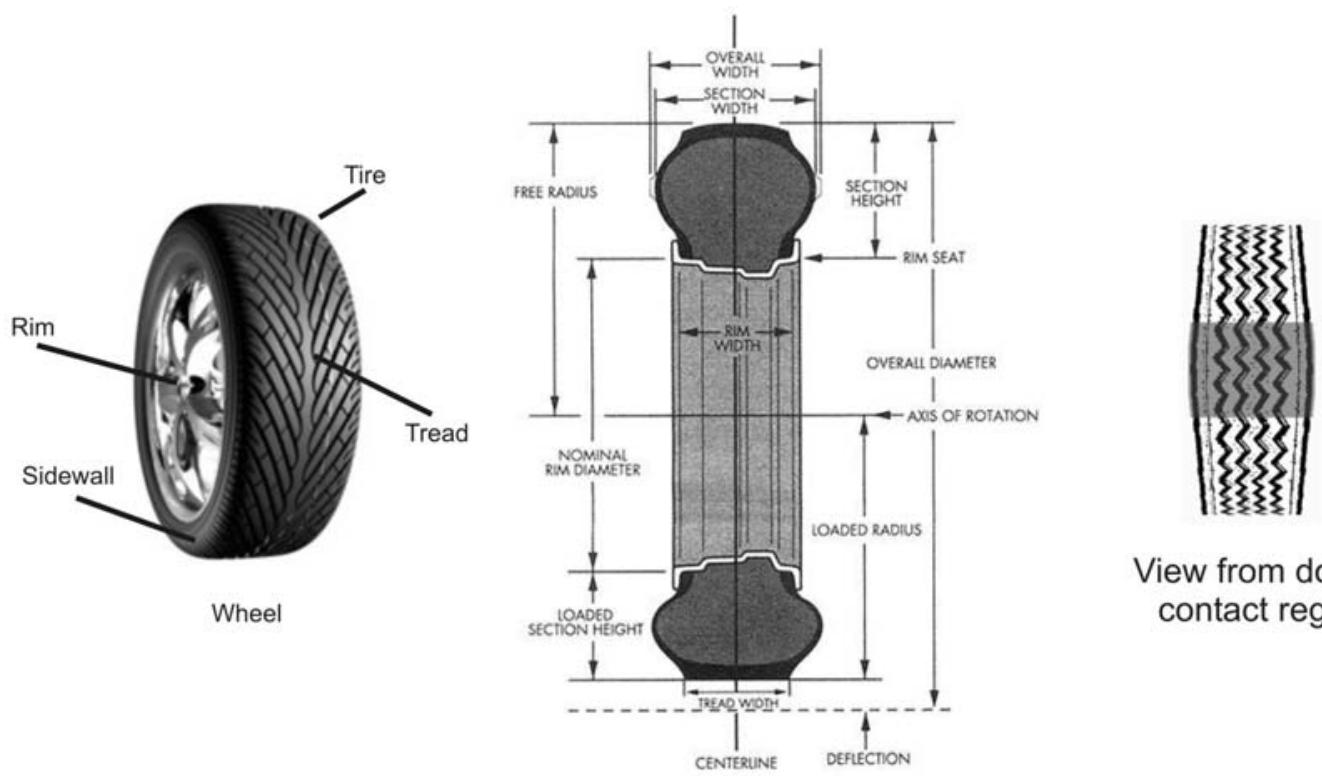

View from down contact region

Figure 1-1 - Actual passengers car wheel, dimensions and contact region

From the engineering viewpoint, if we consider the interaction with the ground, the wheel is not a rigid body - its rim may be considered so, but not the inflated tire: its deformations and corresponding forces must be considered. The iteration between the ground and the tire occurs in the respective contact region. With a vertical load on the wheel, the contact patch is really a squashed oval rather than a rectangle. For the vehicle's usual conditions of ride and cornering, at urban streets or highways in good conditions, we can observe that:

- Under usual wheel's loadings, the contact patch will change in shape and size, but it will continue to be similar to a rectangle or, in more general cases, to a trapezoid.

- As the tire is elastic, the contact region, considered as a plane figure, also will change its relative position to the rim according to the wheel's loading. However, these relative displacements will be small, because all (except the vertical one) are restricted by the friction slide limit (friction coefficient) in the contact: for higher loads, the whole wheel will slide, without increasing 
those relative displacements. Here, "small displacements" mean displacements much smaller than the typical wheel dimensions. Of course, at roads with large holes or very irregular, the contact region will not be even plane anymore, and the relative displacements may be large.

- From a technical viewpoint, these friction coefficients, between rubber and concrete, rubber and asphalt or so, measured under laboratory conditions with clean surfaces, are just reference values. In actual roads, with dust, oil, sand, etc. on them, the real sliding limit may be very different.

- Under usual $4000 \mathrm{~N}$ to $8000 \mathrm{~N}$ vertical load on each wheel, sliding between tire and ground would results in severe tread's wearing. With usual life of 50 $000 \mathrm{~km}$, we may conclude that, with a proper car maintenance, this sliding does not occurs in those usual tire's service conditions. We will show later that the assumption of the occurrence of "micro-sliding”, in some points of the contact region, is not necessary to explain longitudinal and lateral "slips" phenomena in the wheels behavior, at least at the usual conditions considered here. Of course, for extreme accelerations or breaking, or for close curves at high speed, the sliding may (or must) occurs.

Concerning to the tire behavior, from the physical viewpoint, the first observation is that the forces generated from the iteration ground-tire are transmitted to the rim unique and exclusively through the tire's sidewall.

The second observation refers to the sidewall inclination angles in the region in contact with the ground.

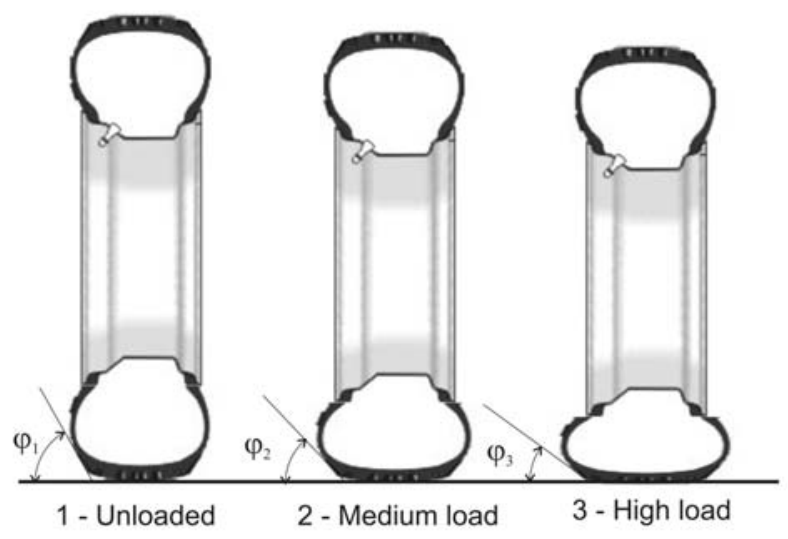

Figure 1-2 - Sidewall angles

As may be seen in Figure 1-2, even if the intensity (modulus) of the force that the sidewall applies to the contact region is constant, its vertical and transversal components may changes significantly with the angle $\varphi$, which is function of the wheel load, or the relative position between the rim and the contact region. It is possible to foresee that the geometric effect of the change of angle $\varphi$ is the main parameter that rules the mechanical behavior of the tire. 


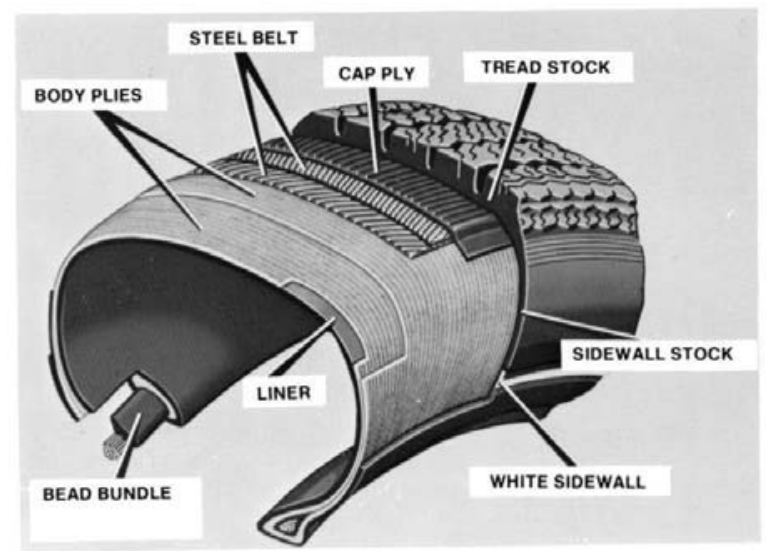

Figure 1-3 - Tire construction

Considering the tire itself, it is made of rubber and a set of fibers and metallic textiles as shown in Figure 1-3, resulting in a anisotropic composite material with different properties in the tread and in the sidewall. The equivalent Young's modulus E' of this composite is very different in compression and tension - the textiles are virtually inextensible in tension and have no effect in compression, in which case the rubber works alone. Many authors considers the composite inextensible in tension, and uses the rubber's E' - about 50 MPa, (EngineeringToolbox, 2013), (PerkinElmer Inc., 2007) - in compression. It is relevant to note that the inflated tire works with a high level of tensile stresses, much higher than the stresses caused by the bending of the tread or of the sidewalls.

\subsection{Tire physical model}

Considering the prior facts, the tire physical model will be built adopting the following hypothesis and simplifications:

1 - The entire tire is considered as a perfectly flexible membrane. It means that bending moments and bending rigidity are neglected. Also, this surface will be considered inextensible in tension, and the rubber mechanical properties will be used in compression and shearing.

2 - The tire is represented by three geometrical surfaces which are defined, as shown in Figure 1-4, by:

a) Two equal and symmetrical sidewalls, (right and left sides), constituted by the partial section of a toroid surface defined by the following dimensions: the external tire radius $(R e)$, its internal radius or the rim radius $(R \mathrm{i})$ and the "curvature parameter" or "bulging parameter" $(c o)$ shown at the Figure 1-5, for the inflated tire without loading. It is also supposed that the rim and the tread have the same width.

b) The tread, also in Figure 1-5, represented by the orthogonal section of a cylinder, is defined by the "equivalent" external radius of the tire $(R e b)$ and the tread's breadth $(L)$. Note: Reb may be a little different from Re but we will consider that $R e$ and Reb coincide. 


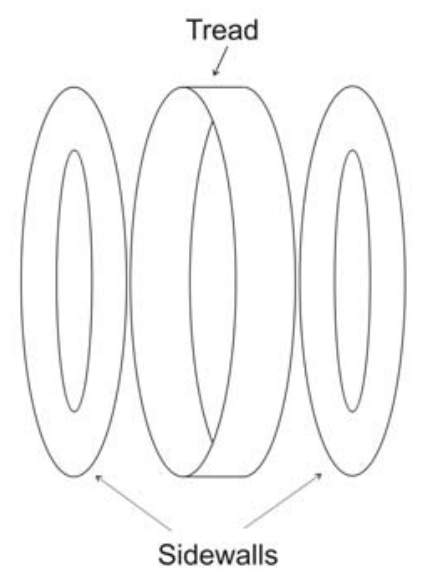

Figure 1-4 - Membrane model

3 - For the sidewalls, the membrane stresses intensity (modulus) is a function of the internal air pressure $p$ and will remain constant under the several loads - just their direction will change. The directions changes will occur according the respective changes of the surfaces geometry, under the corresponding loading cases.

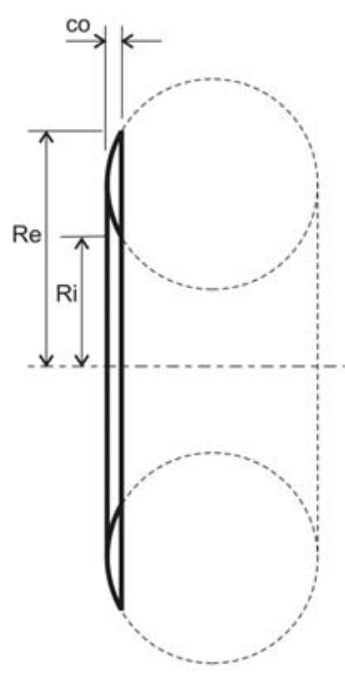

a. One Sidewall

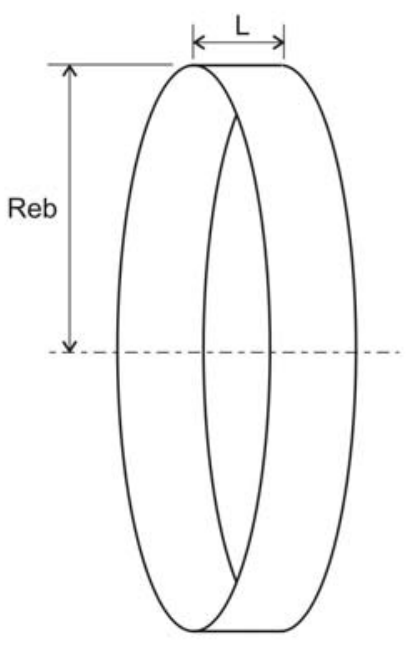

b. Tread

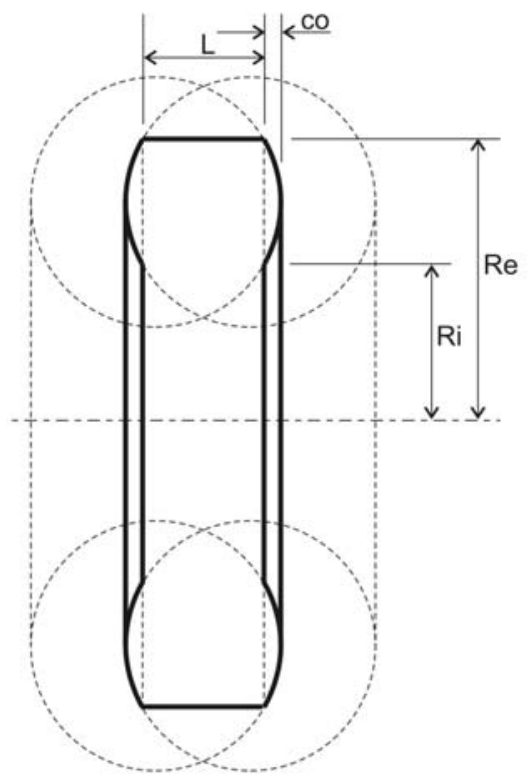

c. Membrane model

Figure 1-5 - Membrane model surfaces

4 - The small changes of the internal gas pressure, due to different loadings, is neglected.

5 - The contact region between the tire and the ground is a plane trapezoid.

6 - There is no sliding (i.e., no relative movement) between tread and ground at the contact points in the contact region. 
7 - Also as a consequence of the previous hypothesis, the condition of "small displacements" will be adopted in several equations development, and indicated in these cases. This approach is analogous to that used in structural engineering, related to beams, plates, shells, etc.. It is usually called "theory of small displacements", and it supposes that the displacements are much smaller than the typical dimensions of the corresponding structural element.

\section{MATHEMATICAL MODEL - GENERAL}

\subsection{Kinematics and coordinates systems}

Consider a fixed horizontal plane and let us define a moving control volume that embraces the contact region between the tire and the ground, as shown in Figure 2-1. For a free wheel rolling on this plane, under vertical only loads, the shape of this region is a rectangle, and let us define the point $C$ of the control volume as the one which coincides with the geometric center of this rectangle in this situation. Let us also define a moving point $\mathrm{O}$, which maintains constant distance $a$ from the plane, and for which $\mathrm{C}$ is its orthogonal projection on the plane. Let us use the Frenet frame $(\vec{t}, \vec{n}, \vec{b})$ of $O$ 's trajectory to define the orthogonal Cartesian coordinates $(O, x, y, z)$, and the corresponding unit vector basis $(\vec{\imath}, \vec{\jmath}, \vec{k})$ by:

$$
\vec{\imath}=\vec{t}
$$

$\vec{\jmath} / / \vec{b}$, positive for the increasing distance from the plane, and

$$
\vec{k}=\vec{\imath} \times \vec{\jmath}
$$

Let us also define a referential $A$ fixed to this coordinates system, which is also fixed to the contact region's control volume.

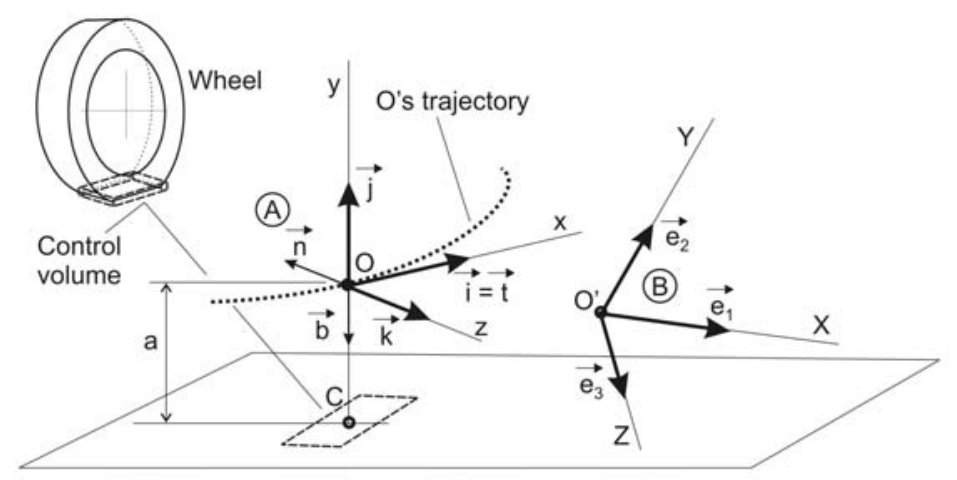

Figure 2-1 Coordinates systems

Also consider the rigid wheel's rim with center $O^{\prime}$, width $L$ and radius $R i$, and another orthogonal Cartesian coordinates $\left(O^{\prime}, X, Y, Z\right)$ with corresponding unit vector basis $\left(\vec{e}_{1}, \vec{e}_{2}, \vec{e}_{3}\right)$. The plane $\left(O^{\prime}, \vec{e}_{1}, \vec{e}_{2}\right)$ always coincides with the rim's middle plane, and this coordinates system $\left(O^{\prime}, X, Y, Z\right)$ defines a referential $B$, as shown in Figure 2-2. The rim's rotation vector, relative to referential $B$, is given by:

$$
\vec{\Omega}=\Omega \vec{e}_{3}
$$




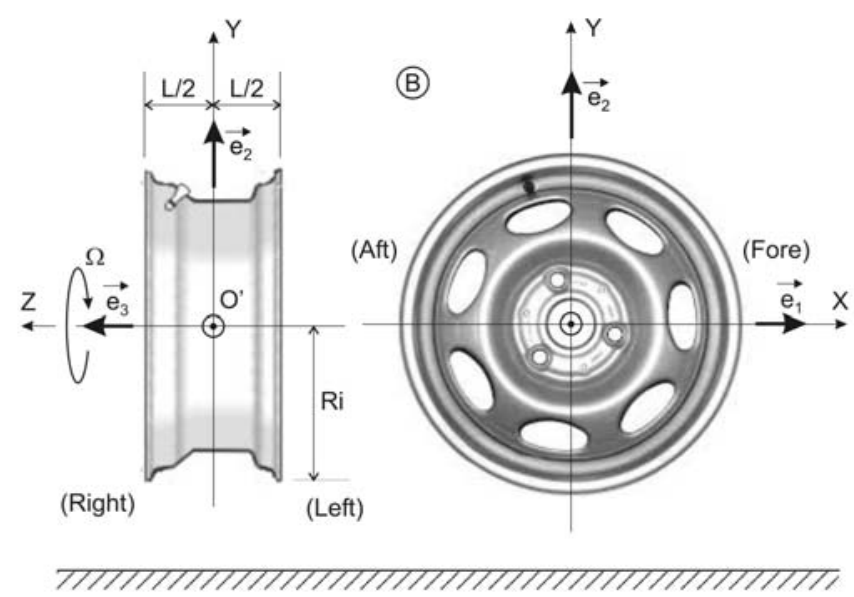

Figure 2-2 - Referential B and rim’s coordinates

For the rotation of the referential $B$, from the initial position where the referential $B$ coincides with referential $A$, let us define a vector $\{\theta\}$ with the Euler angles, in its usual form:

$$
\{\theta\}=\{\phi, \theta, \psi\}
$$

Then, the rotation matrix [Rot] is given by:

$$
[\text { Rot }]=\left[\begin{array}{ccc}
\cos \psi \cos \phi-\cos \theta \sin \phi \sin \psi & \cos \psi \sin \phi+\cos \theta \cos \phi \sin \psi & \sin \psi \sin \theta \\
-\sin \psi \cos \phi-\cos \theta \sin \phi \cos \psi & -\sin \psi \sin \phi+\cos \theta \cos \phi \cos \psi & \cos \psi \sin \theta \\
\sin \theta \sin \phi & -\sin \theta \cos \phi & \cos \theta
\end{array}\right]
$$

and the transformation of coordinates of any vector (position, velocity or acceleration) at the referential $B$, from the coordinates system $B$ to $A$, is given by:

$$
\{x\}_{B}=[R o t]^{t}\{X\}_{B}
$$

\subsection{Forces and displacements}

Suppose an initial situation, where the wheel is rolling on that horizontal plane, free from external forces, except for a vertical load $W_{\mathrm{S}}$ from the vehicle's weight, and with the coordinates systems $A$ and $B$ coinciding. This initial forces system is equivalent to a resultant force $\vec{R}_{S}$, applied to point $O^{\prime}$, and a binary $\vec{M}_{S_{O},}$. The contact region is a rectangle and its control volume does a straight translation in the plane. The rotation vector of the wheel, relative to the referential $\mathrm{B}$, is given by:

$$
\vec{\Omega}=-\Omega \vec{k}
$$

From this initial situation, let us move the wheel's rim (or the referential $B$ ) to a new position, relatively to $A$, displacing its center $O$ ' from the point $O$ and rotating the rim from the frame $(O, x, y, z)$. All are small displacements, as they are limited by the hypothesis of no sliding at the contact region. As consequence, the tire will deform, the contact region may change its shape and size, and the control volume's movement on the plane may be not a straight translation anymore. The control volume's point $C$ will not necessarily coincide with the contact region's geometric center anymore. 
It is necessary to apply a force $\vec{R}_{A}$ and a binary $\vec{M}_{A_{O} \text {, }}$ to the point $O^{\prime}$ of the wheel to achieve this displacement. Neglecting small dynamic effects, these applied forces will be equilibrated by the ground reaction, transmitted by the deformed tire.

Let us consider a steady state situation, where referential B is fixed to A in its new position (and both will move as a single rigid body, in a trajectory to be determined). The tire deformation will remain constant, and the applied forces $\vec{R}_{A}$ and $\vec{M}_{A_{O}}$, in referential $A$, will be also constants.

Concerning to the loads at the interaction tire-ground, the region of the tread in contact with the ground is isolated, and the corresponding force systems are applied to this region, building the so called "free body diagram" of the contact region. It is considered six distributed force systems acting in this region. The whole set of forces, in the absence of inertia forces, constitutes a null system, which implies the static equilibrium of this region. Five of these systems are shown in Figure 2-3 corresponding to the tire forces acting in the contact region. The sixtieth system is the ground reaction on the contact region. From membrane hypothesis, this reaction system is equal and directly opposite to the combined other five ones.

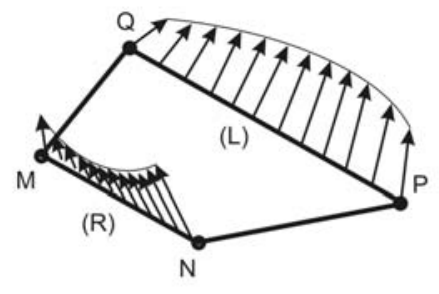

(a) Sidewalls force systems (two - right (R) and left (L))

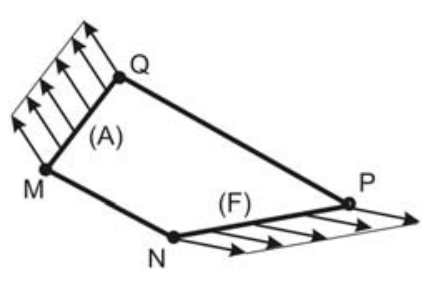

(b) Tread force systems (two - fore (F) and aft (A))

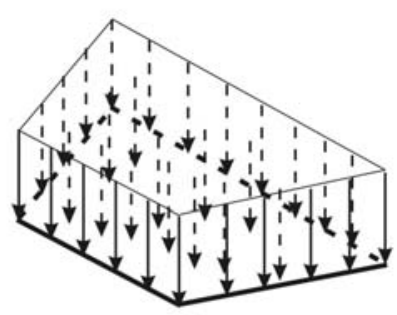

(c) Direct uniform pressure force system

Figure 2-3 - Force systems from the tire, at contact region

From basic mechanics, the system of all distributed forces applied to the contact region by the remaining of the tire, and its internal pressure, is equivalent to a system of one force and one moment $\left(\vec{R} t, \vec{M} t_{C}\right)$, where $\vec{R} t$ is the resultant of those forces, applied to a point $\mathrm{C}$ in the contact region, and $\vec{M} t_{C}$ is the binary equal to the moment of the same forces to pole $C$, as shown in Figure 2-4. Analogously, $\left(\vec{R} g, \vec{M} g_{C}\right)$ are the system equivalent to all the forces from the ground acting in this region. If we neglect dynamic effects, the force systems $\left(\vec{R} t, \vec{M} t_{C}\right)$ and $\left(\vec{R} g, \vec{M} g_{C}\right)$ are directly opposite and we have:

$$
\vec{R} t+\vec{R} g=\overrightarrow{0} \text { and } \vec{M} t_{C}+\vec{M} g_{C}=\overrightarrow{0}
$$




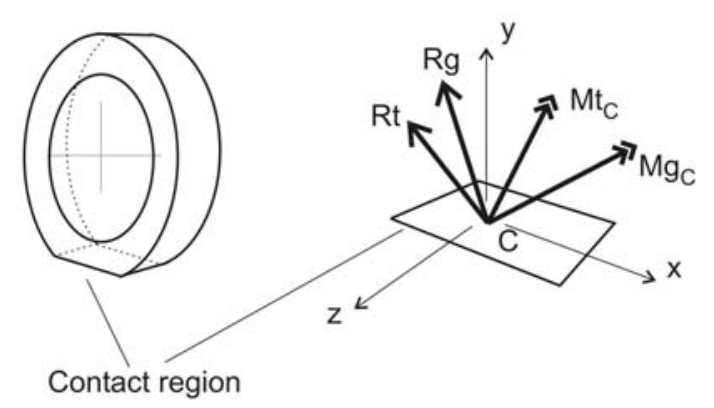

Figure 2-4 - Equivalent forces at contact region

Note that, for the steady state situation, we have also the relationship between the ground forces and the forces applied to the rim:

$$
\vec{R} g+\vec{R}_{S}+\vec{R}_{A}=\overrightarrow{0} \text { and } \vec{M} g_{C}+\vec{M}_{S_{O},}+\vec{M}_{A_{O},}+\left(C-O^{\prime}\right) \times\left(\vec{R}_{S}+\vec{R}_{A}\right)=\overrightarrow{0}
$$

We have five force systems at the contact region, as shown in Figure 2-3. If we use $k$ to designate each of them:
$k=1$ : Right Left sidewall force system;
$k=2$ : Left sidewall force system;
$k=3$ : Fore tread force system;
$k=4:$ Aft tread force system;
$k=5$ : Direct pressure force system,

Let us also represent the force system $\left(\vec{R} t, \vec{M} t_{O}\right)$, where $\vec{M}_{t_{O}}=\vec{M} t_{C}+(C-O) \times \vec{R}_{t}$, by a single vector $\{R\}$, with this five force systems:

$$
\{R\}=\left\{\begin{array}{c}
R t_{x} \\
R t_{y} \\
R t_{z} \\
M t_{O x} \\
M t_{O y} \\
M t_{O z}
\end{array}\right\}=\left\{\begin{array}{l}
F_{1} \\
F_{2} \\
F_{3} \\
F_{4} \\
F_{5} \\
F_{6}
\end{array}\right\}=\left\{R^{1}\right\}+\left\{R^{2}\right\}+\left\{R^{3}\right\}+\left\{R^{4}\right\}+\left\{R^{5}\right\}=\sum_{k=1}^{k=5}\left\{R^{k}\right\}
$$

where $F_{i}$ for $\mathrm{i}=1,2$ and 3 are the resultant force components in respectively $x, y$ and $z$ directions. Also, $F_{i}$ for $\mathrm{i}=4,5$ and 6 are the resultant moment components relative to the center of the wheel, about axis $x, y$ and $z$, respectively, and $\left\{R^{k}\right\}$ stands for the force system $k$.

For small displacements, the new rim's position, in the coordinates system $A$, may be defined by a unique vector $\{\delta\}$ with six components:

$$
\{\delta\}=\left\{\begin{array}{l}
\delta_{1} \\
\delta_{2} \\
\delta_{3} \\
\delta_{4} \\
\delta_{5} \\
\delta_{6}
\end{array}\right\}
$$


where $\delta_{\mathrm{i}}, \mathrm{i}=1,2,3$, correspond to the linear displacements of point $\mathrm{O}^{\prime}$ along the axis $O x$, $O y$ and $O z$, respectively, and $\delta_{\mathrm{i}}, \mathrm{i}=4,5,6$, correspond to the rotations (small angular displacements) of the rim, about the same axis.

These relative displacements between the reference systems $A$ and $B$, and the corresponding tire deformation, are shown in Figure 2-5.
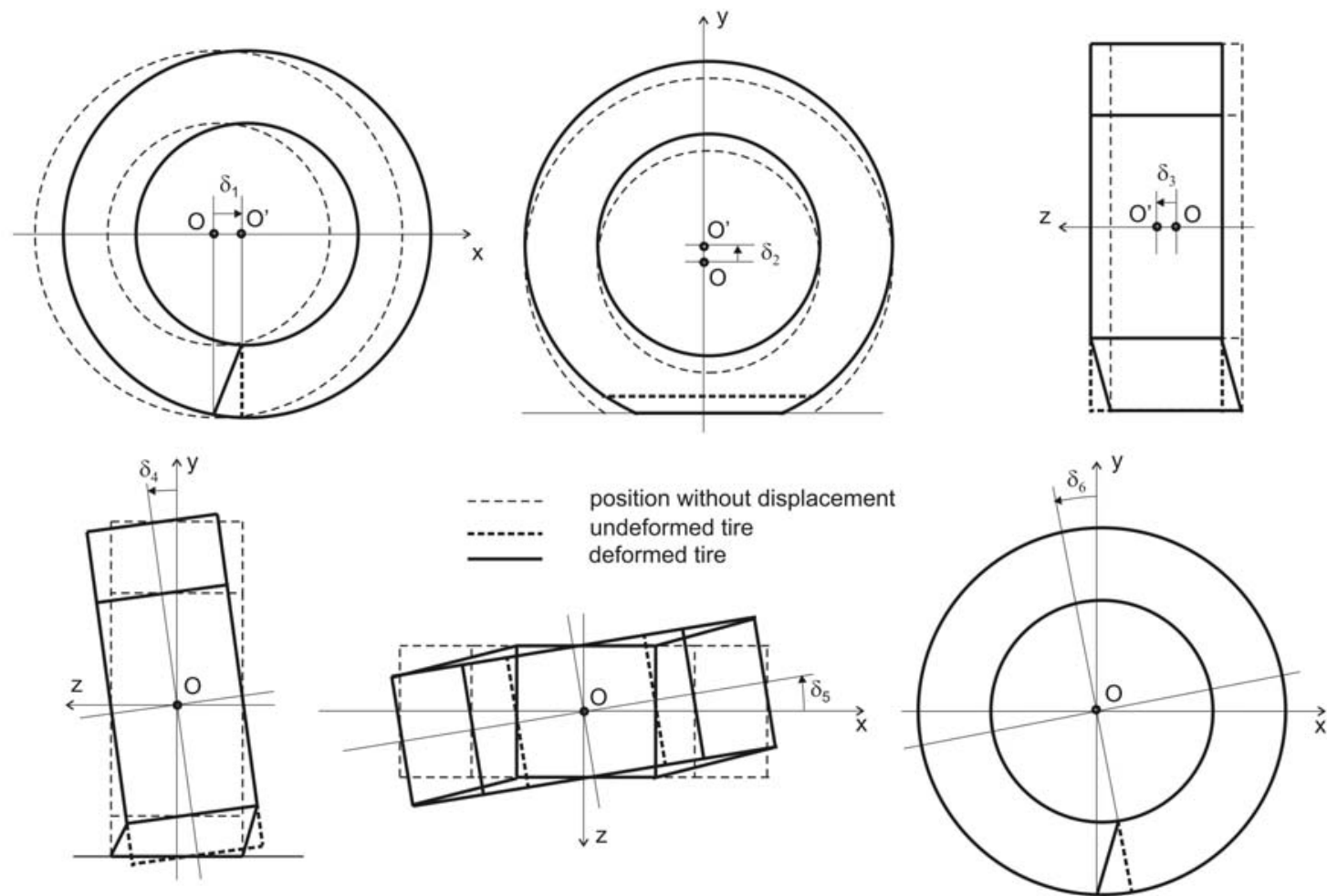

Figure 2-5 - Relative small displacements $\delta_{\mathrm{i}}$ and tire deformation

Each $\{R\}_{k}$ is a function of the rim's displacement, i.e.:

$$
\left\{R^{k}\right\}=\left\{R^{k}\left(\delta_{1}, \delta_{2}, \delta_{3}, \delta_{4}, \delta_{5}, \delta_{6}\right)\right\}
$$

These forces arise from the relative displacement of the rim to the contact region. From the assumption of small displacements, we may neglect coupling effects and write:

$$
\begin{gathered}
\left\{R^{k}\right\}=\left\{R_{0}^{k}\right\}+\left\{R_{1}^{k}\left(\delta_{1}\right)\right\}+\left\{R_{2}^{k}\left(\delta_{2}\right)\right\}+\left\{R_{3}^{k}\left(\delta_{3}\right)\right\}+\left\{R_{4}^{k}\left(\delta_{4}\right)\right\}+\left\{R_{5}^{k}\left(\delta_{5}\right)\right\}+ \\
\left\{R_{6}^{k}\left(\delta_{6}\right)\right\}=\left\{R_{0}^{k}\right\}+\sum_{j=1}^{6}\left\{R_{j}^{k}\left(\delta_{j}\right)\right\}
\end{gathered}
$$

where $\left\{R_{0}^{k}\right\}$ is the force vector for null relatives displacement, and $\left\{R_{j}^{k}\left(\delta_{j}\right)\right\}$ is the change in the force vector due to relative displacement $\delta_{j}$.

Also, for small displacements, we may use the Taylor's expansion, neglecting higher order terms, and obtain a linear approximation for the relationship between forces and displacements: 
where

$$
\left.\left\{R_{j}^{k}\left(\delta_{j}\right)\right\} \cong \frac{d}{d \delta_{j}}\left\{R_{j}^{k}\left(\delta_{j}\right)\right\}\right|_{\delta_{j}=0} \cdot \delta_{j}=\left\{R_{j}^{k^{\prime}}\left(\delta_{j}\right)\right\} \cdot \delta_{j}
$$

and, then:

$$
\left\{R_{j}^{k^{\prime}}\left(\delta_{j}\right)\right\}=\left.\frac{d}{d \delta_{j}}\left\{R_{j}^{k}\left(\delta_{j}\right)\right\}\right|_{\delta_{j}=0}
$$

$$
\left\{R^{k}\right\}=\left\{R_{0}^{k}\right\}+\sum_{j=1}^{6}\left\{R_{j}^{k \prime}\left(\delta_{j}\right)\right\} \cdot \delta_{j}
$$

Now we may write the relationship between forces and displacements as:

$$
\left\{R^{k}\right\}=\left\{R_{0}^{k}\right\}+\left[\left\{R_{j}^{k^{\prime}}(0)\right\}^{t}\right]\{\delta\}=\left\{R_{0}^{k}\right\}+\left[F^{k}\right]^{t}\{\delta\}
$$

where

$$
F_{i j}^{k}=R_{i j}^{k \prime}(0)
$$

\section{CONCLUSION}

This text presented an initial approach to physical and mathematical model of a automotive tire. Next, we will present the expressions of $F_{i j}^{k}$ for each $\delta_{j}$, assuming that all other $\delta_{n}=0$, for $n \neq j$, in a text that will be published soon.

\section{REFERENCES}

BÖHM, F. 1966. Mechanik des Gürtelreifens. Ingenieur Archiv 35. 1966, pp. 82-101.

CarBibles. 2011. Car Bibles: The Wheel and Tyre Bible. [Online] 2011. http://www.carbibles.com/tyre_bible.html.

EngineeringToolbox. 2013. Elastic Properties and Young Modulus for some Materials. The Engineering Toolbox. [Online] 2013. http://www.engineeringtoolbox.com/youngmodulus-d_417.html.

KUNG, L.E., SOEDEL, W. and YANG, T.Y. 1986. Free-vibration of a pneumatic tire-wheel unit using a ring on an elastic-foundation and a finite-element model. Journal of Sound and Vibration. June 8, 1986, pp. 181-194.

LECOMTE, C., GRAHAM, W.R. and DALE, M. 2010. A shell model for tyre belt vibration. Journal of Sound and Vibration. May 10, 2010, pp. 1717-1742.

MERIAN, J. L. and KRAIGE, L. G. 2008. Engineering Mechanics: Statics. USA : Wiley, 2008.

NAKASHIMA, H. and WONG, J.Y. 1993. A 3-dimensional tire model by the finite-element method. Journal of Terramechanics. 1993, Vol. 30, 1, pp. 21-34.

PACEJKA, H. B. and SHARP, R. S. 1991. Shear force development by pneumatic tires in steady-state conditions - a review of modeling aspects. Vehicle System Dynamics. 1991, Vol. 20, 3-4, pp. 121-175.

PACEJKA, H. B. 2006. Tire and vehicle dynamics. 2nd. s.l. : SAE International, 2006.

—. 2002. Tyre and Vehicle Dynamics (R372). s.l. : SAE International, 2002. R372. 
PerkinElmer Inc. 2007. Characterization of Car Tire Rubber. USA :

http://www.perkinelmer.com/CMSResources/Images/44-

74057APP_CharacterizationOfCarTireRubber.pdf, 2007.

PISSARENKO, G. S., IAKOVLEV, A. P. and MATVEIEV, V. V. 1985. Prontuário de resistência de materiais. Moscow, URSS : MIR Ed., 1985.

Salvagni, Ronaldo B., Alves, Marcelo A. L. and Barbosa, Roberto S. 2013. A Physical and Mathematical Model for Inflated Tires. (to be published). 2013.

SHARP, R.S. and EL-NASHAR, M.A. 1986. A Generally Applicable Digital Computer Based Mathematical Model for the Generation of Shear Forces by Pneumatic Tyres. Vehicle System Dynamics. 1986, Vol. 15, 4, pp. 187-209.

TIMOSHENKO, S. P. and GOODIER, J. N. 1970. Theory of Elasticity. 3rd. s.l. : McGraw Hill, 1970.

TIMOSHENKO, S. P. and WOINOWSKY-KRIEGER, S. 1964. Theory of Plates and Shells. 2nd. s.l. : McGraw Hill, 1964.

VIL'KE, V. G. and DVORNIKOV, M. V. 1998. The rolling of a wheel with a pneumatic tire on a plane. J. Appl. Maths Mechs. 1998, Vol. 62, 3, pp. 359-369.

VIL'KE, V. G. and KOZHEVNIKOV, I. F. 2004. The rolling of a wheel with a reinforced tire along a plane with slipping. J. Appl. Maths Mechs. 2004, Vol. 68, pp. 909-921.

—. 2001. The rolling of a wheel with a reinforced tire along a plane without slip. J. Appl. Maths Mechs. 2001, Vol. 65, 6, pp. 915-927.

YONG, R.N., FATTAH, E.A. and BOONSINSUK, P. 1978. Analysis and prediction of tiresoil interaction and performance using finite elements. J. Terramechanics. 1978, Vol. 15, 1, pp. 43-63.

YOUNG, W. and BUDYNAS, R. 2001. Roark's Formulas for Stress and Strain. 7th. s.l. : McGraw-Hill, 2001.

ZACHOW, D. 1997. 3D membrane shell model in application of a tractor and PKW tyre. VEHICLE SYSTEM DYNAMICS. 1997, Vol. 27, Suppl. S, pp. 163-172. 